\title{
Isolation and characterization of stem cells from differentially degenerated human lumbar zygapophyseal articular cartilage
}

\author{
LIANG XIAO $^{1 *}$, SHUJUAN XU ${ }^{2 *}$, XIAO WANG ${ }^{1}$, ZHONGXING JIN $^{1}$, \\ JING WANG $^{1}$, BIJING YANG ${ }^{1}$ and HONGGUANG XU ${ }^{1}$ \\ Departments of ${ }^{1}$ Orthopedic Surgery and ${ }^{2}$ Surgery, Yijishan Hospital, \\ The First Affiliated Hospital of Wannan Medical College, Wuhu, Anhui 241001, P.R. China
}

Received March 27, 2018; Accepted September 28, 2018

DOI: $10.3892 / \mathrm{mmr} .2018 .9592$

\begin{abstract}
The present study aimed to verify the presence of stem cells with multilineage differentiation potential in human lumbar zygapophyseal articular cartilage (LZAC) and to compare the chondrogenic potential of cells obtained from differentially degenerated articular cartilage samples. Surgically obtained human lumbar zygapophyseal joint tissues were classified into the normal, mildly degenerated and severely degenerated groups, according to their pathological characteristics. Primary chondrocytes from these groups were cultured, and stem cells were selected using a monoclonal cell culture method. Differences in stem cell morphology between the three groups were observed using inverted microscopy and phalloidin staining. In addition, stem cell chondrogenic potential was determined through induced differentiation and cellular staining. Gene and protein expression levels of the chondrogenic-specific markers aggrecan, collagen type-II and SRY-related high-mobility-group box 9 were determined using reverse transcription-quantitative polymerase chain reaction and western blotting. The clonogenic ability of stem cells in the three groups was determined using a clonogenic assay. It was revealed that stem cells with multilineage differentiation potential were isolated from all three cartilage groups; however, the cells obtained from severely degenerated articular cartilage resulted in severe fibrosis, whilst those obtained from mildly degenerated articular cartilage possessed stronger chondrogenic and clonogenic abilities. Taken together, stem cells with multilineage differentiation potential and clonal properties were
\end{abstract}

Correspondence to: Professor Hongguang $\mathrm{Xu}$, Department of Orthopedic Surgery, Yijishan Hospital, The First Affiliated Hospital of Wannan Medical College, 2 Zheshan West Road, Wuhu, Anhui 241001, P.R. China

E-mail: pumchxuhg@126.com

${ }^{*}$ Contributed equally

Key words: lumbar zygapophyseal joint, osteoarthritis, cartilage, degeneration, stem cells identified in human LZAC, and these characteristics were more prominent in mildly degenerated as compared with severely degenerated articular cartilage.

\section{Introduction}

Lower back pain (LBP) is a common disorder with quick recovery observed in the majority of patients. However, the rate of LBP recurrence is high and adversely affects the quality of life of the patients (1). Studies have indicated that LBP can arise following lumbar facet joint osteoarthritis (LFJOA), which accounts for $15-45 \%$ of non-specific LBP cases (2). Facet joints are paired zygophyseal joints between two consecutive vertebrae. LFJOA is intimately linked to the distinct but functionally associated condition of degenerative disc disease, which affects structures in the anterior aspect of the vertebral column. The prevalence of facet-mediated pain in clinical populations increases with increasing age, suggesting that LFJOA may have a particularly important role in older adults with spinal pain.

Over the past two decades conceptualization of knee OA has shifted away from a predominant focus on cartilage degeneration towards a view of OA as a heterogeneous and dynamic process of whole-joint failure resulting from an imbalance between the breakdown and repair of joint tissues. However, clinical treatment of LFJOA is currently restricted to conservative management, including medicine administration (such as nonsteroidal anti-inflammatory drugs) and physiotherapy. Surgical intervention can be introduced when the disorder is complicated by lumbar degenerative disease. Direct resection of the zygapophyseal joint and bone graft fusion is common; however, this approach cannot fully cure LFJOA or restore function. An optimal LFJOA treatment should not only eliminate pain and remove the cause of pain, but also restore and maintain the normal structure and physiological function of the lumbar zygapophyseal joint (LZJ).

The application of stem cells in cartilage tissue repair is developing rapidly, providing a potential new therapeutic approach for LFJOA $(3,4)$. However, the presence of stem cells within the lumbar zygapophyseal articular cartilage (LZAC) has yet to be confirmed. The repair and regeneration of LFJOA cartilage may be achieved through a single injection of lab-grown LZAC to provide a cell source with multilineage 
differentiation potential combined with a cell scaffold biomaterial and/or cartilage differentiation-inducing factors.

The present study aimed to investigate mesenchymal stem cell (MSC)-like cells isolated from human LZAC and to compare their differentiation potentials in differentially degenerated articular cartilage tissues. The findings are expected to further the current understanding on stem cell restoration and LFJOA therapy.

\section{Materials and methods}

Patient data and inclusion criteria. The present study was approved by the Ethics Committee of Yijishan Hospital, The First Affiliated Hospital of Wannan Medical College (Wuhu, China). A total of 36 LZJ tissue samples were obtained during surgery performed on patients with lumbar degenerative disease between March 2017 and August 2017. The patient age ranged between 27 and 71 years, and the most commonly affected surgical segments were L4-S1. The inclusion criteria were as follows: i) Patients who were clinically diagnosed with lumbar spinal stenosis, spondylolisthesis and degenerative lumbar scoliosis, exhibited little or no curative effects following conservative management and volunteered to undergo surgery (5); ii) patients without symptoms of rachiterata or spinal cord tumors; iii) the surgery included posterior lumbar decompression, bone fusion and vertebral pedicle internal fixation. Written informed consent was obtained from all enrolled patients. All the subsequent research analyses were carried out in accordance with the approved guidelines.

Pathological staining of the LZJ. The collected LZJ samples were fixed in $10 \%$ formalin for $48 \mathrm{~h}$, cut into 2-mm slices along the coronal plane, decalcified for 1 week, embedded in paraffin and sectioned. The $5 \mathrm{~mm}$ sections were then dewaxed, dehydrated and air-dried prior to staining with hematoxylin/eosin (HE) and Oil Red O (Gibco; Thermo Fisher Scientific, Inc., Waltham, MA, USA). Tissue morphology was observed using an inverted microscope.

The samples were classified according to the Osteoarthritis Cartilage Histopathology Assessment System of the Osteoarthritis Research Society International and the classifying methods detailed by Kim et al (6). Samples with an intact articular cartilage surface and tissue structures were assigned to the normal group (G0). Samples with discontinuities or fissures on the articular cartilage surface, mild loss of mesochondrium and/or chondrocyte responsive hypertrophy were included in the mildly degenerated group (G1-G2). Finally, samples with defects or deformations of the articular cartilage surface, severe loss of mesochondrium and/or a significantly decreased cartilage cell number were assigned to the severely degenerated group (G3-G4).

Cultivation of primary chondrocytes and isolation of stem cells. LZJ articular cartilage was isolated from the joint surface under sterile conditions, cut into $1-\mathrm{mm}^{3}$ sections using ophthalmic scissors and digested for $4-5 \mathrm{~h}$ at $37^{\circ} \mathrm{C}$ using collagenase type-II (0.25\%; Gibco; Thermo Fisher Scientific, Inc.). Following digestion, cell suspensions were filtered twice through a 200-mesh stainless steel filter and centrifuged at $1,000 \mathrm{x} g$ for $5 \mathrm{~min}$ at $37^{\circ} \mathrm{C}$ to obtain the cell pellet. Subsequent to the removal of the supernatant, Dulbecco's modified Eagle's medium/Ham's F12 (DMEM/F12; Gibco; Thermo Fisher Scientific, Inc.) supplemented with $15 \%$ fetal bovine serum (FBS; Gibco; Thermo Fisher Scientific, Inc.) was used to disperse the pellet by pipetting, and then cells were seeded into a $10-\mathrm{cm}$ culture plate at a density of $100 \mathrm{cells} / \mathrm{cm}^{2}$. Cells were incubated at $5 \% \mathrm{CO}_{2}$ and $37^{\circ} \mathrm{C}$, and the medium was changed every 3 days until monoclonal cells were observed. Subsequent experiments demonstrate that the monoclonal cells have various properties of stem cells.

Induced differentiation and cell staining. Primary stem cells were seeded into 24 -well plates at a density of $2 \times 10^{4}$ cells/well in $1 \mathrm{ml} \mathrm{DMEM} / \mathrm{F} 12$ supplemented with $10 \% \mathrm{FBS}$. When the cells reached $80 \%$ confluence, the medium was replaced with osteogenic, adipocytic or chondrogenic differentiation medium (Gibco; Thermo Fisher Scientific, Inc.). The differentiation media were changed every 3 days. After induction for 2-3 weeks, cells were fixed in $4 \%$ paraformaldehyde solution at $25^{\circ} \mathrm{C}$ for $40 \mathrm{~min}$ and washed with PBS. Cells were then stained with $500 \mu \mathrm{l}$ alizarin red S $(0.4 \%)$, Oil Red O or safranin $\mathrm{O}$ (Gibco; Thermo Fisher Scientific, Inc.) to identify the osteogenic, adipogenic or chondrogenic differentiation, respectively. Following incubation at $25^{\circ} \mathrm{C}$ for $1 \mathrm{~h}$, the staining solutions were removed, and the samples were washed with PBS, prior to observation and imaging using an inverted phase-contrast microscope.

Flow cytometry. Isolated primary stem cells were washed with PBS, digested in trypsin $(0.25 \%$; Gibco; Thermo Fisher Scientific, Inc.) and placed into Eppendorf tubes $(2 \mathrm{ml})$. Phycoerythrin-conjugated mouse anti-human monoclonal antibodies against CD14 (eBioscience; Thermo Fisher Scientific, Inc.; 11-0149-41; 1:50), CD90 (eBioscience; Thermo Fisher Scientific, Inc.; 11-0909-41; 1:50), CD105 (eBioscience; Thermo Fisher Scientific, Inc.; 12-1057-41; 1:50), CD73 (eBioscience; Thermo Fisher Scientific, Inc.; 11-0739-41; 1:50), CD45 (eBioscience; Thermo Fisher Scientific, Inc.; 11-9459-41; 1:50), CD34 (eBioscience; Thermo Fisher Scientific, Inc.; 11-0349-41; 1:50), STRO-1 (eBioscience; Thermo Fisher Scientific, Inc.; 14-6688-82; 1:50) and HLA-DR (eBioscience; Thermo Fisher Scientific, Inc. 11-9956-42; 1:50) were then added. IgG (mouse IgG1 $\kappa$ isotype control-FITC, eBioscience; Thermo Fisher Scientific, Inc.; 11-4714-81; 1:20; mouse IgG1 $\kappa$ isotype control-PE; eBioscience; Thermo Fisher Scientific, Inc.; 12-4714-41, 1:100; mouse IgG2b $\kappa$ isotype control-PerCP-Cyanine5.5; eBioscience; Thermo Fisher Scientific,Inc.; 45-4732-80; 1:100) were used as isotype control. Subsequent to mixing, the samples were incubated for $30 \mathrm{~min}$ in the dark $25^{\circ} \mathrm{C}$, washed with PBS, re-suspended in $500 \mu \mathrm{l}$ PBS and analyzed using flow cytometry (BD Biosciences, San Diego, CA, USA). Threshold values were set using negative cell lines in accordance with the fluorescence intensities of isotype controls. Positive expression rates and fluorescence intensities against each of the aforementioned monoclonal antibodies were detected using duplicate samples.

Cytoskeleton staining. Primary stem cells were seeded into 6-well plates at a density of $2 \times 10^{5}$ cells/well. The cells were fixed in $4 \%$ paraformaldehyde at $25^{\circ} \mathrm{C}$ for $15 \mathrm{~min}$ and 
permeabilized using $0.3 \%$ Triton $\mathrm{X}-100$ ( $2 \mathrm{ml} /$ well $)$ for $10 \mathrm{~min}$ at $37^{\circ} \mathrm{C}$. The cells were then washed with PBS and $100 \mu \mathrm{l} /$ well phalloidin (Gibco; Thermo Fisher Scientific, Inc.) was added prior to incubation for $10 \mathrm{~min}$ in the dark $25^{\circ} \mathrm{C}$. DAPI $(100 \mu \mathrm{l})$ was subsequently added and the cells were incubated for 5 min in the dark $25^{\circ} \mathrm{C}$. Following washing in PBS, cytoskeletal F-actin was observed and imaged using laser-scanning confocal microscopy (Zeiss LSM 510-META laser scanning microscope; Carl Zeiss AG, Jena, Germany).

Reverse transcription-quantitative polymerase chain reaction $(R T-q P C R)$. RNA was extracted from the induced cells using TRIzol ${ }^{\circledR}$ reagent (Invitrogen; Thermo Fisher Scientific, Inc.). The quantity and quality of isolated RNA were evaluated using absorbance at wavelengths of 260 and $280 \mathrm{~nm}$. Reverse-transcribed into cDNA using a RevertAid ${ }^{\mathrm{TM}}$ First Strand cDNA Synthesis kit (Thermo Fisher Scientific, Inc.) according to the manufacturer's protocol of the kit. Next, qPCR was performed using a QuantiTect SYBR Green PCR kit (Thermo Fisher Scientific, Inc.), according to the manufacturer's protocol, and the mRNA levels of aggrecan (ACAN), collagen type-II (COL-II) and SRY-related high-mobility-group box 9 (SOX9) were measured in each group. The conditions of real-time PCR were as follows: Denaturation at $95^{\circ} \mathrm{C}$ for $10 \mathrm{sec}, 40$ cycles at $95^{\circ} \mathrm{C}$ for $10 \mathrm{sec}$ and $60^{\circ} \mathrm{C}$ for $30 \mathrm{sec}$. Dissociation curves revealed no nonspecific amplification. The primers used for qPCR assay are listed in Table I. GAPDH was used as an internal reference, and the results were analyzed using the $2^{-\Delta \Delta \mathrm{Cq}} \operatorname{method}(7)$.

Western blot analysis. Induced cells were collected, resuspended in RIPA Lysis buffer (Invitrogen; Thermo Fisher Scientific, Inc.) with phenylmethanesulfonyl fluoride (PMSF; Thermo Fisher Scientific, Inc.) and lysed on ice. The supernatants were collected, and protein concentrations were measured using the BCA method. Equal amounts of proteins were used in subsequent experiments. A total of $20 \mu \mathrm{g}$ protein was loaded per lane using 10\% SDS-PAGE and electrotransferred onto nitrocellulose membranes (Beyotime Institute of Biotechnology, China), and 5\% bovine serum albumin (Beyotime Institute of Biotechnology) was used for membrane blocking for $1 \mathrm{~h}$ at $25^{\circ} \mathrm{C}$. Primary antibodies against ACAN (1:1,000; Abcam, Cambridge, UK), COL-II (1:5,000; Abcam), SOX9 (1:5,000; Abcam) and GAPDH (1:5,000; Abcam) were incubated with the membrane overnight at $4^{\circ} \mathrm{C}$ on a shaker. Following washing, the membrane was exposed to a secondary antibody diluted at 1:5,000 in blocking reagent and incubated for $1 \mathrm{~h}$ at room temperature on a shaker. Subsequent to further washing in Tris-buffered saline/20\% Tween-20, the membranes were visualized using an enhanced chemiluminescence detection system (EMD Millipore, Billerica, MA, USA). Immunoreactive bands were quantified on autoradiography films in triplicate with using Image $\mathbf{J}$ software (version 4.8; National Institutes of Health, Bethesda, MD, USA) by normalizing the band intensities to GAPDH.

Colony-forming assay. Primary stem cells were seeded into 6 - $\mathrm{cm}$ diameter culture dishes at a density of 100 cells/dish $(n=3$ per sample). After 7-10 days of cultivation, cells were fixed in $4 \%$ paraformaldehyde at $25^{\circ} \mathrm{C}$ for $20 \mathrm{~min}$, washed with PBS
Table I. Primer sequences for quantitative polymerase chain reaction.

\begin{tabular}{ll}
\hline Gene & \multicolumn{1}{c}{ Primer sequence $\left(5^{\prime}-3^{\prime}\right)$} \\
\hline ACAN & F: CATTCACCAGTGAGGACCTCGT \\
& R: TCACACTGCTCATAGCCTGCTTC \\
COL-II & F: TGAGGGCGCGGTAGAGACCC \\
& R: TGCACACAGCTGCCAGCCTC \\
SOX9 & F: ATCTGAAGAAGGAGAGCGAG \\
& R: TCAGAAGTCTCCAGAGCTTG \\
GAPDH & F: GCACCGTCAAGGCTGAGAAC \\
& R: TGGTGAAGACGCCAGTGGA \\
\hline
\end{tabular}

ACAN, aggrecan; COL-II, collagen type-II; SOX9, SRY-related high-mobility-group box 9; F, forward; R, reverse.

and immersed in $0.1 \%$ crystal violet staining solution at $25^{\circ} \mathrm{C}$ for $10 \mathrm{~min}$. Following washing with PBS, the cell colonies were counted and imaged.

Statistical analysis. All statistical data are expressed as the mean \pm standard deviation. Statistical analysis was conducted using SPSS version 18.0 software (SPSS, Inc., Chicago, IL, USA). Statistically significant differences among the three groups were determined using one-way analysis of variance, and $\chi^{2}$ tests were used to compare cell counts between groups. $\mathrm{P}<0.05$ was considered to indicate a difference that was statistically significant.

\section{Results}

Pathological and radiological characteristics of differentially degenerated LZAC. HE and safranin O staining were performed to classify $36 \mathrm{LZJ}$ articular tissues into three groups according to the degree of degeneration, as follows: i) Normal group $(n=5)$; ii) mildly degenerated group $(n=12)$; and iii) severely degenerated group $(n=19)$. In the normal group, the articular cartilage surfaces were smooth, and the four-layered tissue structures were intact. By contrast, the articular cartilage surfaces of the mildly degenerated group were rough and exhibited longitudinal fissures, while the separation lines of the four-layered structure were unclear. In the severely degenerated group, erosion and defects were clearly observed, with a thinner cartilaginous layer and evident proliferation of osseous tissue. Furthermore, the boundary between the subchondral bone and underlying bone tissues was unclear in this group (Fig. 1A and B).

Cross-sectional computed tomography scans of the lumbar area were also compared among the three groups, and the radiological characteristics for each group were in agreement with the staining results (Fig. 1C). In addition, no significant differences were detected in the clinical data among the three patient groups (Table II).

Stem cells from differentially degenerated LZAC display MSC-like characteristics. Subsequent to osteogenic 
Table II. Comparison of clinical characteristics among the three groups $(n=36)$.

\begin{tabular}{lcccc}
\hline Group & N & Sex (male/female) & Age (years) & Chronic disease (yes/no) \\
\hline Normal & 5 & $3: 2$ & $48.20 \pm 12.07$ & $3: 2$ \\
Mildly degenerated & 12 & $8: 4$ & $53.25 \pm 7.71$ & $6: 6$ \\
Severely degenerated & 19 & $13: 6$ & $55.26 \pm 9.69$ & $10: 9$ \\
F/ $\chi^{2}$ & & 0.124 & 1.126 & 0.143 \\
P-value & 0.940 & 0.337 & 0.931 \\
\hline
\end{tabular}

A

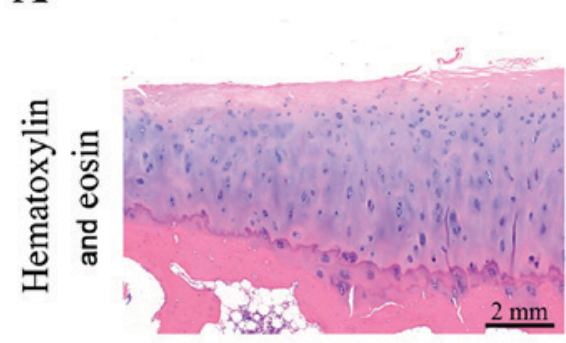

B
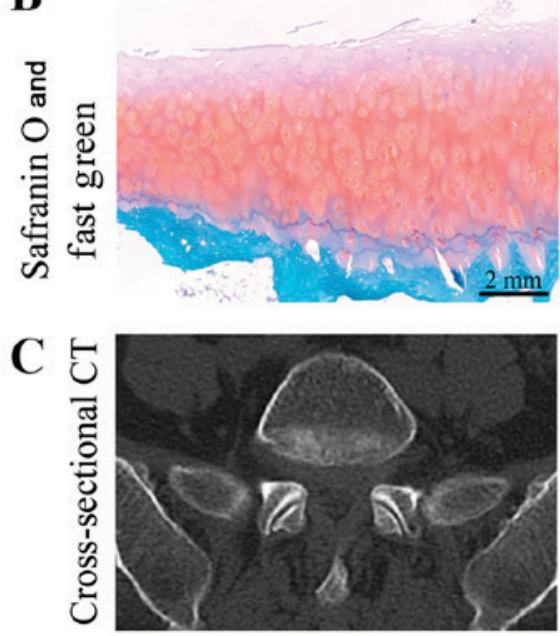

Mildly degenerated
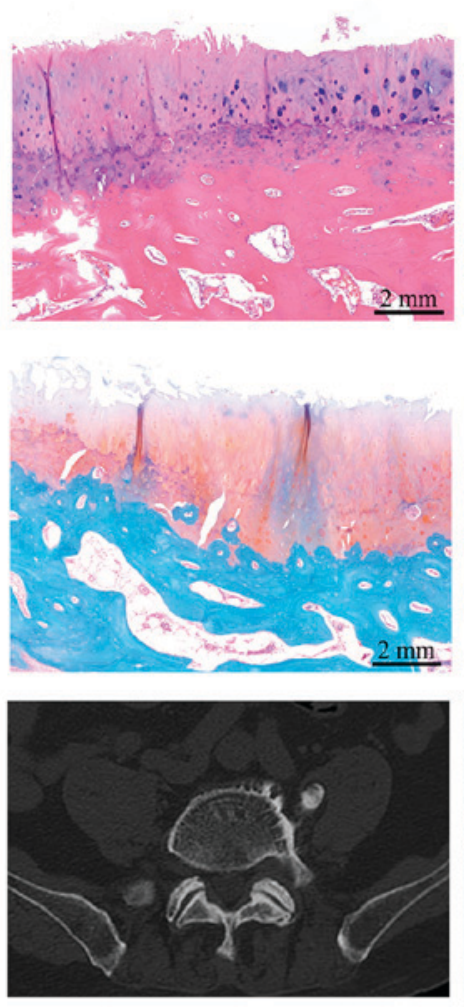

\section{Severely degenerated}
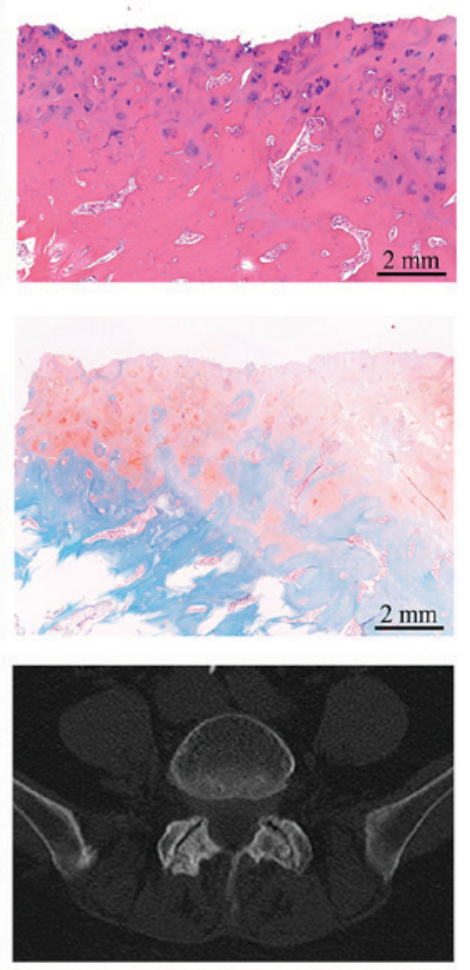

Figure 1. (A) Hematoxylin \& eosin and (B) safranin $\mathrm{O}$ and Fast Green staining revealed severe depletion of proteoglycans, fibrillation, and structural and morphological changes in mildly degenerated (G1-G2) and severely degenerated (G3-G4) lumbar zygapophyseal articular cartilage, compared with normal cartilage (G0). (C) Cross-sectional computed tomography images of lumbar areas revealed similar pathological and radiological characteristics. CT, computed tomography.

differentiation of isolated stem cells, rock salt gradually deposited and formed a plurality of granular calcium nodules. Alizarin red staining result indicated all stem cells were stained red (Fig. 2A). After adipogenic induction, Oil Red O staining results demonstrated that 3 kinds of stem cells were all stained red (Fig. 2B). Chondrocyte-specific aggrecan were stained red with safranin $\mathrm{O}$ following chondrogenic induction (Fig. 2C). Flow cytometry analysis further revealed that isolated stem cells from all groups were positive for the CD90, CD73, STRO-1 and CD105 surface markers, and negative for CD14, CD34, CD45 and HLA-DR (Fig. 3).

Morphological changes are observed in stem cells from differentially degenerated LZACs. Primary chondrocytes from the normal and mildly degenerated groups were polygonal and tightly arranged, while those from the severely degenerated group were elongated and loosely arranged. Stem cells isolated from monoclones were different to primary chondrocytes; more specifically, they were smaller in size and arranged in a whirlpool configuration. No evident differences in the morphology were observed between the three cell groups microscopically. As presented in Fig. 4, phalloidin staining revealed that the stem cell cytoskeletons in the normal group were spindle-shaped, while those in the mildly and severely degenerated groups were visibly elongated. These results indicated that, compared with normal LZAC, abnormal fibrosis was evident in stem cells isolated from mildly and severely degenerated LZAC cases.

Chondrogenic ability differs among stem cells isolated from differentially degenerated LZAC. RT-qPCR and western blot analysis confirmed that stem cells from the mildly 

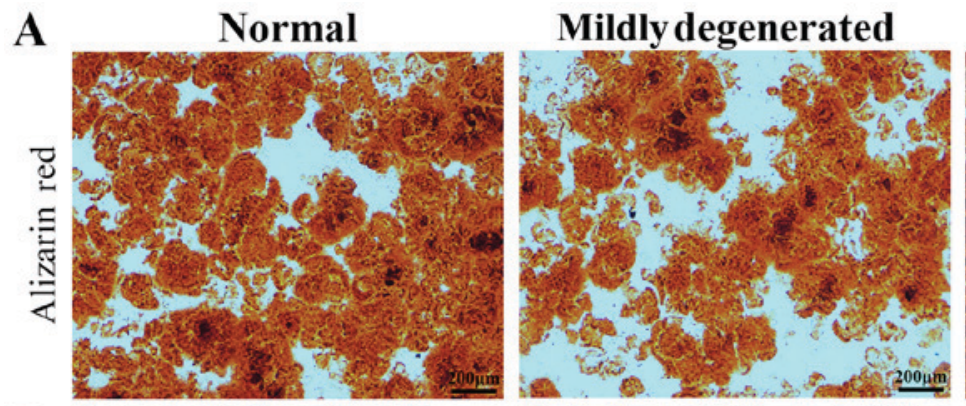

\section{Severely degenerated}
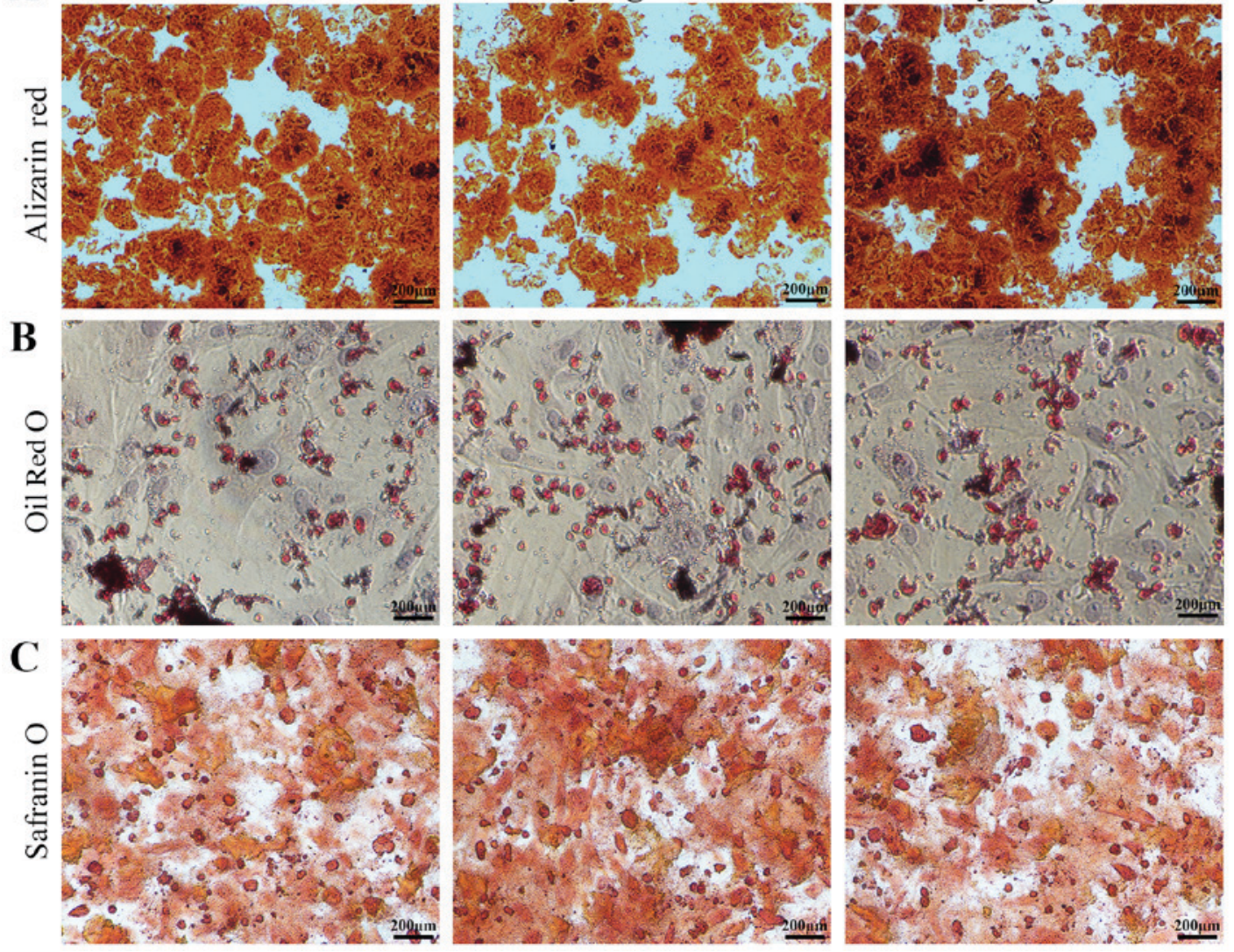

Figure 2. (A) Osteogenic, (B) adipogenic and (C) chondrogenic differentiation, examined by alizarin red S, Oil Red O and safranin O staining. Stem cells from human lumbar zygapophyseal articular cartilage in the three differentially degenerated groups had same differentiation potential to three lineages with 14-21 days of induction. Original magnification: x100.
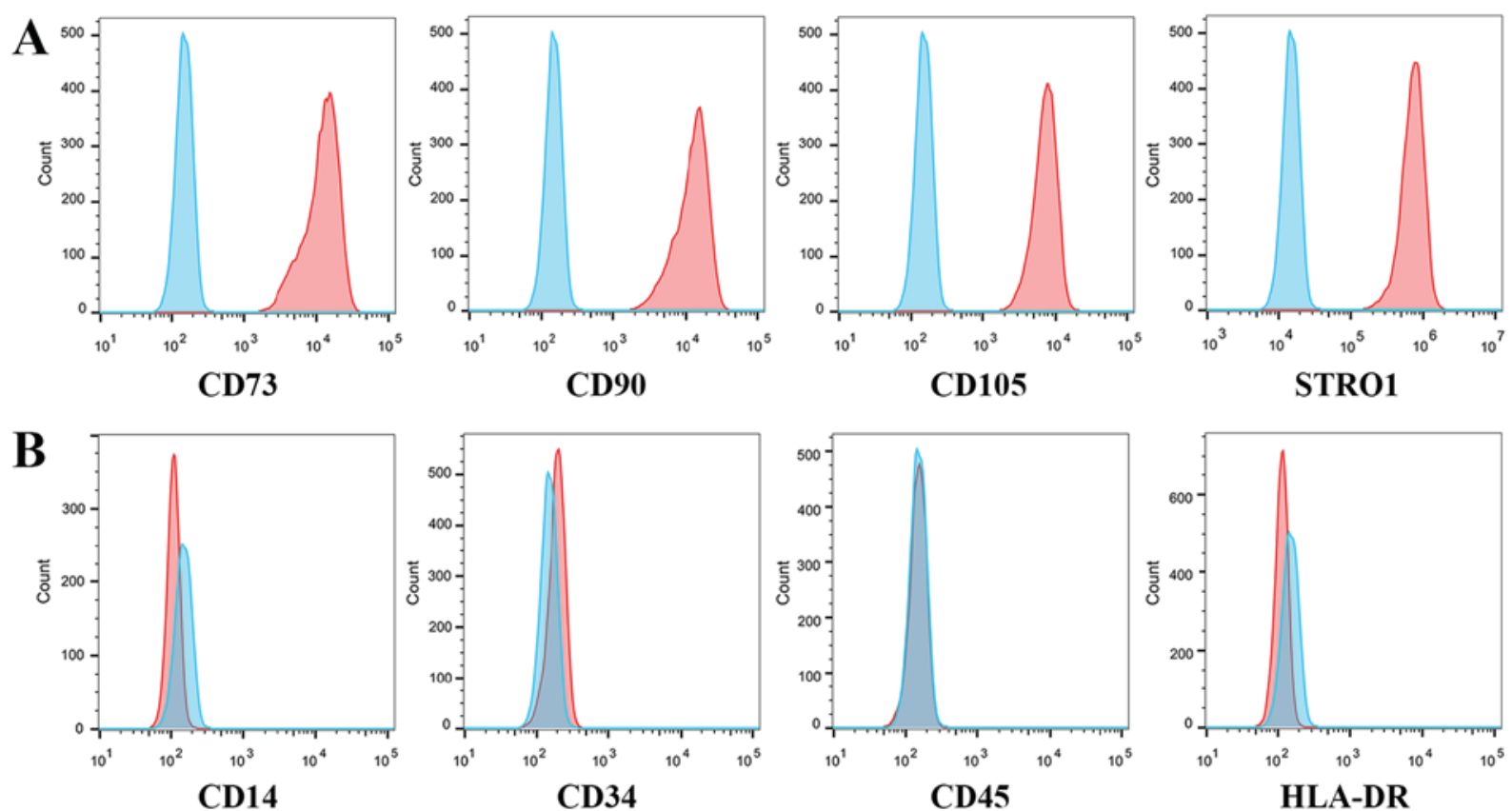

Figure 3. Stem cells from all groups were: (A) Positive for the typical MSC marker proteins CD73, CD90, CD105 and STRO-1; and (B) negative for surface marker expression, including hematopoietic progenitor cell marker CD34, leukocyte common antigen CD45, the immune marker CD14, and HLA-DR. Blue graphs indicated isotype controls, red graphs indicated positive cells. MSC, mesenchymal stem cell; CD, cluster of differentiation.

degenerated group had enhanced chondrogenic potential, whereas those from the severely degenerated group had reduced chondrogenic potential, compared with cells from the normal group. The chondrogenic differentiational genes 
A

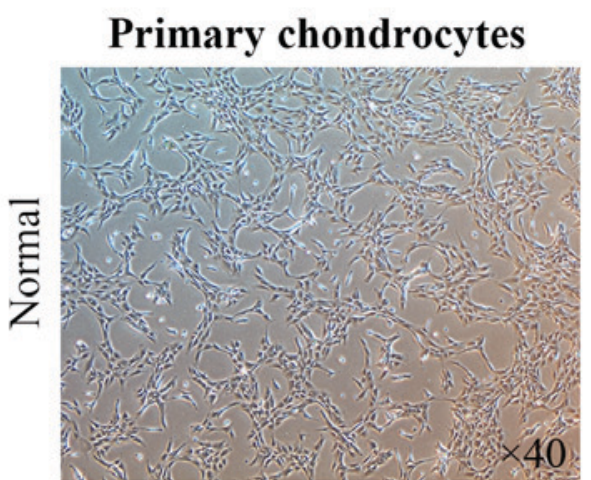

B

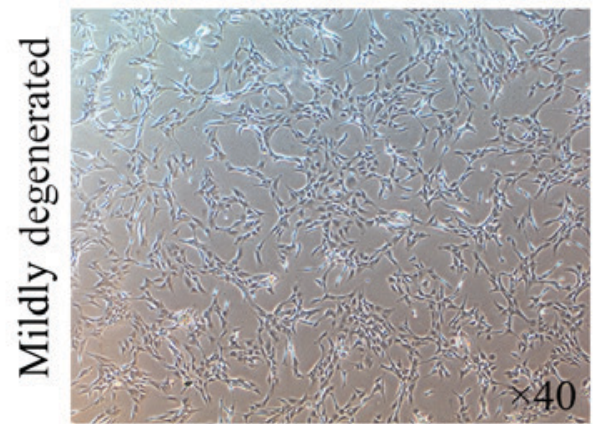

C

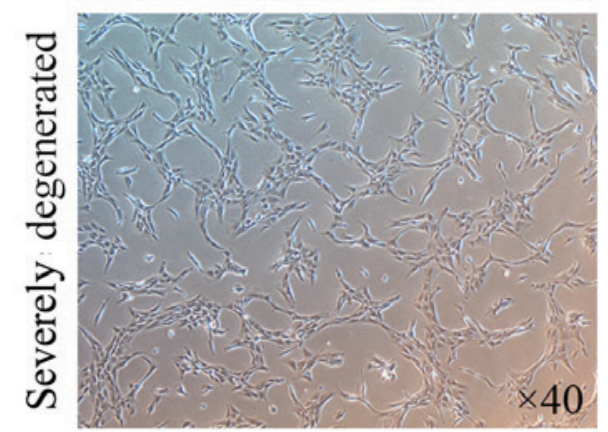

Stem/progenitor cells
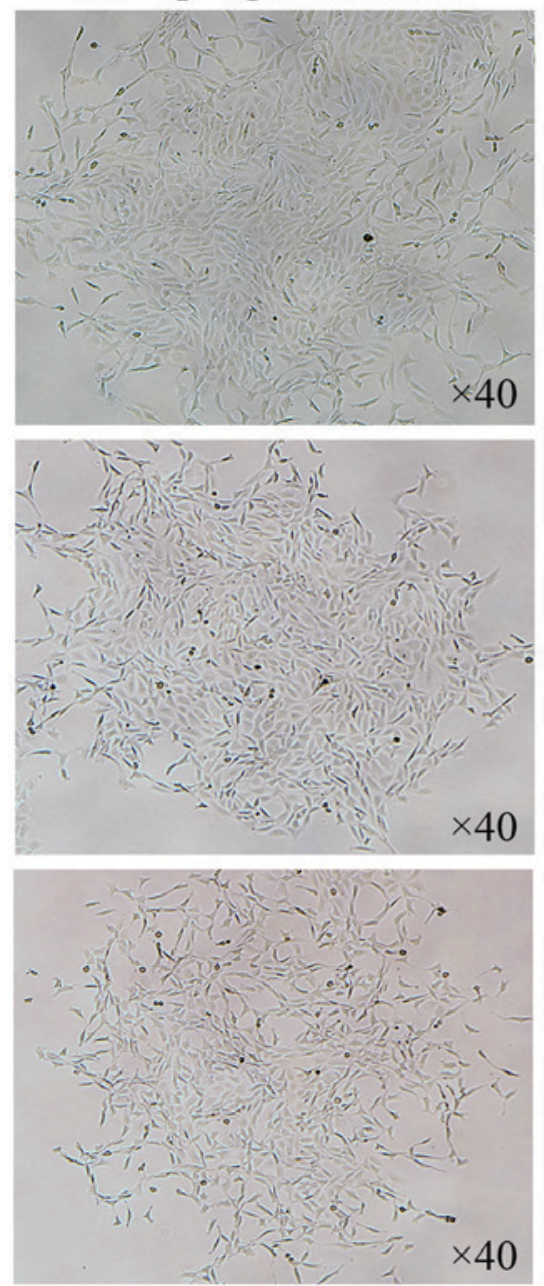

Cytoskeletons of stem/progenitor cells
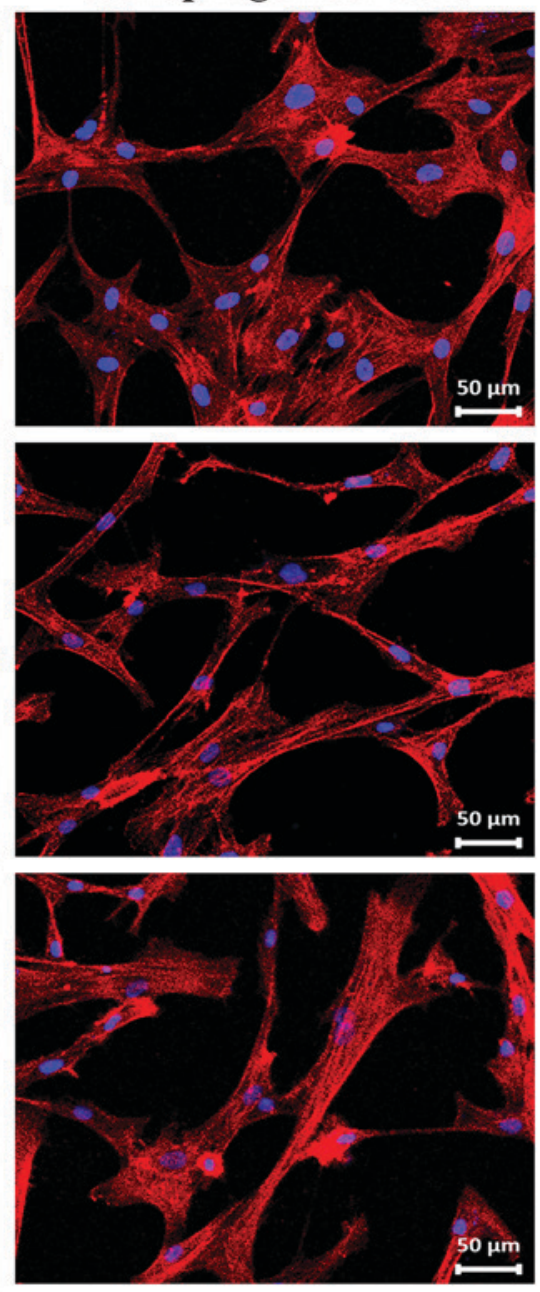

Figure 4. Microscopy results in the (A) normal, (B) mildly degenerated and (C) severely degenerated groups. Microscopy revealed regular cell morphology in the normal group. Stem cells isolated from the mildly degenerated group were elongated, although the morphology of primary chondrocytes was normal. Primary chondrocytes and stem cells from the severely degenerated group were visibly fiberized. Original magnification: $\mathrm{x} 40$.

and proteins (ACAN, COL-II and SOX9) of 3 kinds of stem cells were analyzed respectively, and the results demonstrated that the expression of the 3 genes and proteins by the mildly degenerated group was stronger than the other two cells. And the 3 genes and proteins expression of the normal group was the second highest and that of the severely degenerated group was worse (Fig. 5). These results indicated that, although stem cells with multilineage differentiation potential existed in LZAC at different degrees of degeneration, stem cells from mildly degenerated LZAC exhibited a stronger chondrogenic potential.

Stem cell clonal formation varies in differentially degenerated LZAC. Stem cells isolated from all three groups were observed to form colonies; however, the colony number was significantly lower in the severely degenerated group. No significant difference was observed between the normal and mildly degenerated groups with respect to colony formation (Fig. 6). These results indicated that stem cells from normal and mildly degenerated LZAC possess good clonal formation ability.

\section{Discussion}

Osteoarthritis is characterized by an imbalance between the dynamic processes of cartilaginous tissue decomposition and repair (8). Similarly, the main pathogenic manifestations of LFJOA include abrasion of the arthrodial cartilage, responsive hypertrophy of the subchondral bone and osteophyte formation in the joint verge $(9,10)$. Histological evaluation of osteoarthritis has confirmed increased levels of chondrocyte apoptosis, loss of proteoglycans secreted by chondrocytes and a denser arrangement of chondrocytes in degenerated cartilaginous tissues (11).

Cartilaginous tissue has no blood vessels or nerves, and is composed of 5\% chondrocytes and $95 \%$ mesochondrium; thus, it has a poor self-repairing and regenerative capacity (12). Regenerative medicine has become a focal point, allowing regenerating of defective cartilaginous tissues using autologous chondrocytes (13-15). However, the application of chondrocytes in repair strategies has a number of limitations, including the limited availability of adult chondrocytes. Chondrocytes are terminally differentiated cells with an extremely weak 


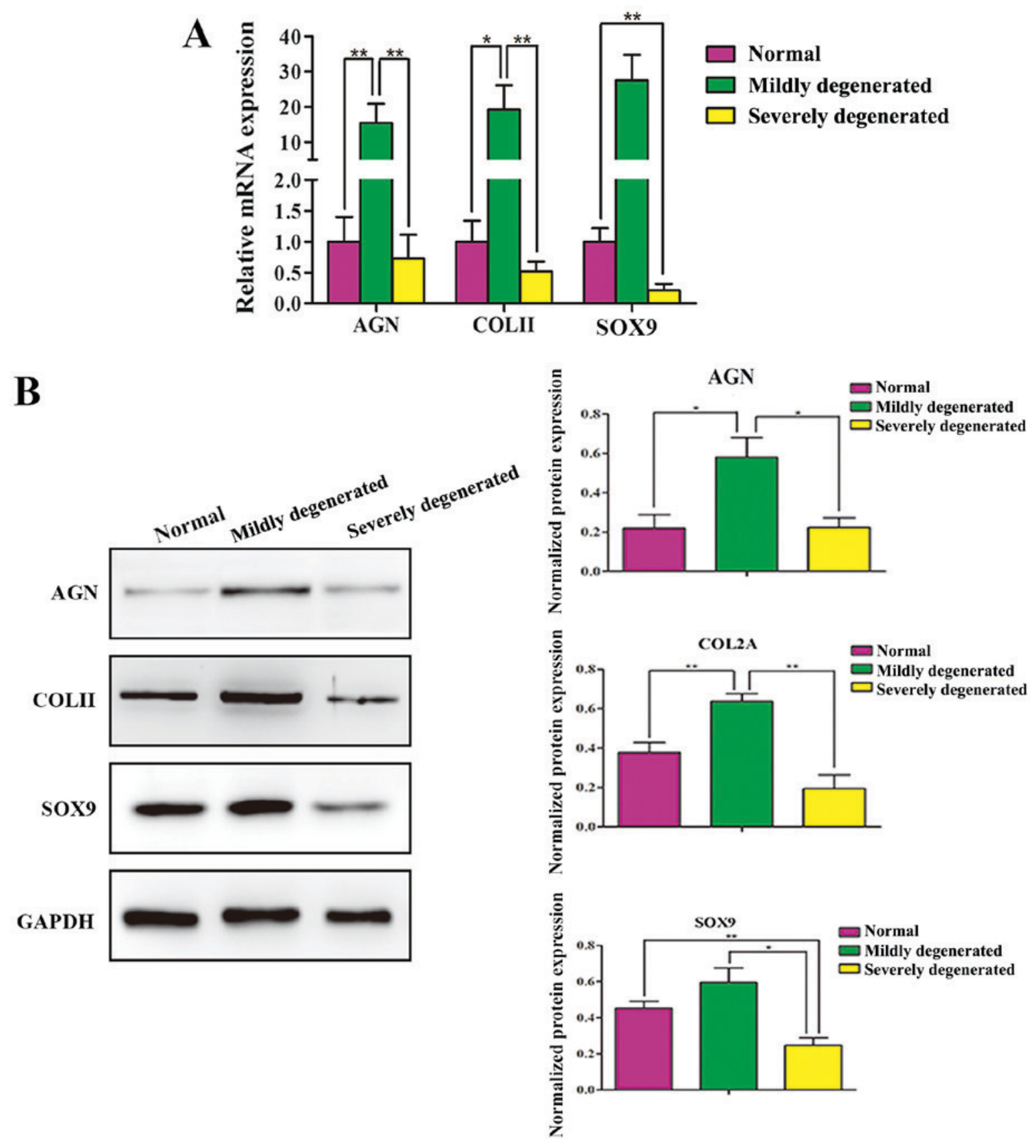

Figure 5. mRNA and protein expression levels of ACAN, COL-II and SOX9 were higher in the mildly degenerated group following chondrogenic induction, as determined by (A) reverse transcription-quantitative polymerase chain reaction analysis and (B) western blot analysis. " $\mathrm{P}<0.05$ and ${ }^{* *} \mathrm{P}<0.01$. ACAN, aggrecan; COL-II, collagen type-II; SOX9, SRY-related high-mobility-group box 9.

proliferative capacity, and dedifferentiation often occurs during in vitro cultivation. Furthermore, the chondrogenic potential of chondrocytes is lost in vitro, making it difficult to obtain a large number of normally functioning chondrocytes from a small number of adult chondrocytes (16-18). Additionally, the extraction efficiency of primary chondrocytes from cartilaginous tissues is low (19).

Research in this field is currently focusing on the repair of bone and cartilage using stem cells. Studies have demonstrated that cells with differentiation capacities exist in both normal and degenerated cartilage $(20,21)$. Clinical tests have verified that implanting or injecting MSCs can promote cartilage formation in articulatio genus defect areas (22). Although MSC isolation from the endplate and LZAC remains under investigation, the future application of these stem cells within spinal fusion surgery and for degenerative disc disease is promising (23-26).

In the present study, the monoclonal cell culture method was used to prove the existence of stem cells with clonal ability and multidifferentiation potential in human LZAC. As part of the LZA microenvironment, these cells have vital roles in the initiation and progression of degenerative spinal diseases, and thus may be a more desirable cell source for tissue repair.

Although stem cells with self-renewal and multilineage differentiation capacities have been found to exist in both normal and degenerated articular cartilage, their full potential for application in tissue repair remains unclear. A number of previous studies have suggested that stem cells only exist on 

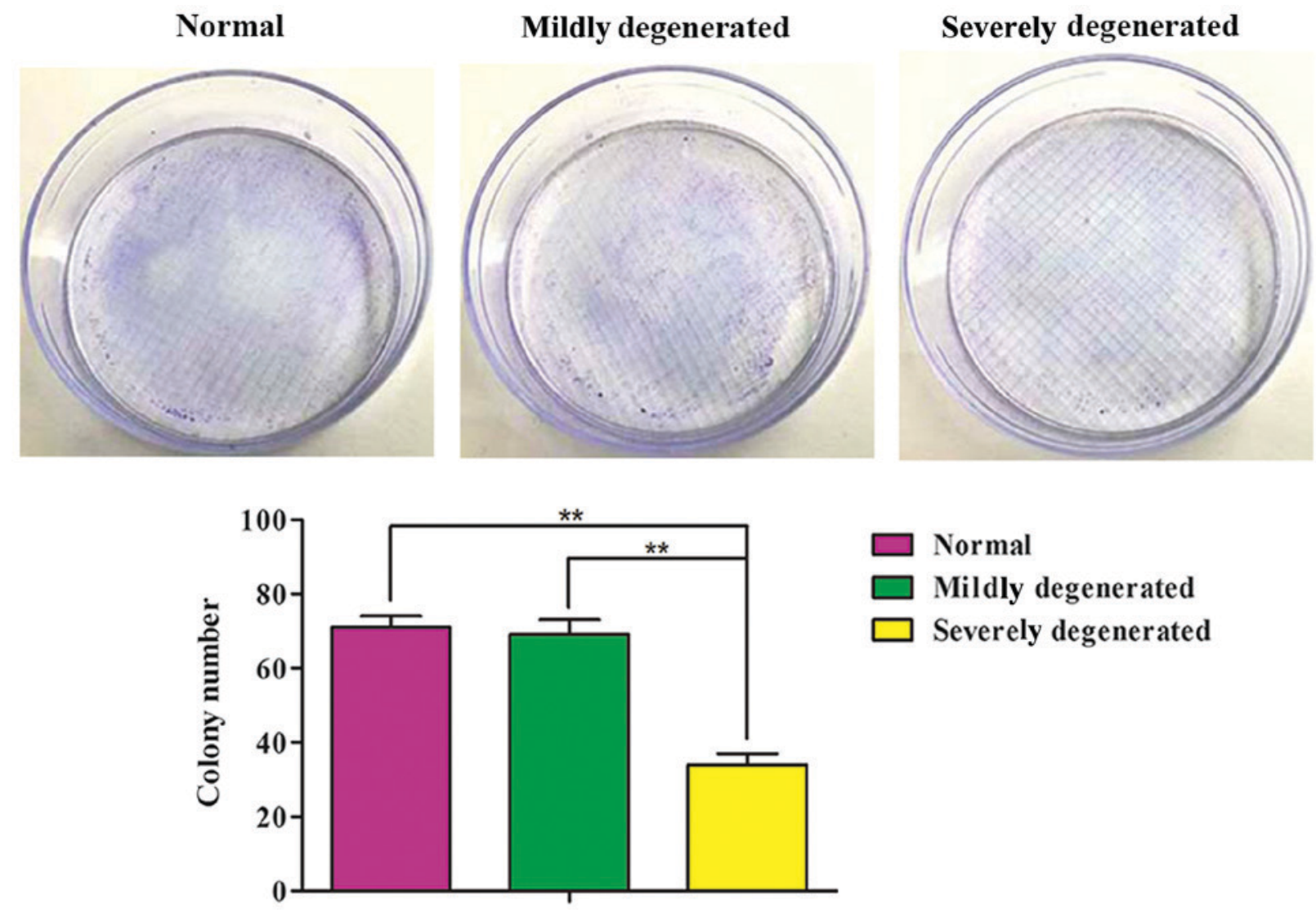

Figure 6. Crystal violet staining revealed a significantly reduced number of colonies in the severely degenerated group. No significant difference was observed between the normal and mildly degenerated groups. ${ }^{* *} \mathrm{P}<0.01$.

surfaces where the cartilage is rapidly growing and developing, while others propose that stem cells are more prevalent at the boundary area of cartilage injury $(27,28)$. These studies indicate, to a certain extent, that stem cells are necessary for the supplementation and maintenance of mesochondrial components. Conversely, the differentiation of stem cells also depends on the extracellular microenvironment (29). In the present study, although stem cells with induced differentiation and clonogenic abilities were isolated from differentially degenerated LZAC, their chondrogenic differentiation and clonogenic abilities differed significantly among the groups.

Cytoskeleton staining revealed fibrosis in the stem cells isolated from mildly and severely degenerated LZAC, indicating that the mesochondrial environment altered the characteristics of these cells to some extent $(30,31)$. Subsequent experiments suggested that stem cells isolated from mildly degenerated LZAC exhibited enhanced chondrogenic differentiation and clonogenic abilities compared with those from normal LZAC. Furthermore, stem cells from severely degenerated LZAC exhibited reduced chondrogenic differentiation and clonogenic abilities.

The present study successfully isolated stem cells from differentially degenerated LZAC using a monoclonal cell culture method, and compared the chondrogenic and clonogenic characteristics in the different groups. However, there are certain limitations: i) The osteogenic and adipogenic differentiation capacities of stem cells require further investigation, as do their surface marker profiles, in comparison with the well-characterized bone marrow-derived MSCs; and ii) although the differentiation potential of stem cells from cartilage tissue is affected by multiple factors, induction models based on nutrition, mechanics and inflammation were not established or investigated in the present study.

As the stem cells were obtained from differentially degenerated LZAC tissues, it is hypothesized that the stem cells from mildly degenerated articular cartilages are in a 'transitional' state, and that their increased differentiation and clonal abilities are likely to be transiently enforced due to external stimuli. During the aggravation of cartilage tissue degeneration, stem cells gradually lose their normal functionality. These findings are important for specific clinical treatment of differentially degenerated cartilage in the future.

In conclusion, stem cells with multilineage differentiation potential and clonal properties were identified in human LZAC, and these characteristics were more prominent in mildly degenerated, compared with severely degenerated, articular cartilage.

\section{Acknowledgements}

Not applicable.

\section{Funding}

The present study was supported by the National Natural Science Foundation of China (grant no. 81572185), the Natural Science Foundation of Anhui Province of China (grant no. 1708085MH185) and the Natural Science Foundation 
for Young Scientists of Anhui Province of China (grant no. 1808085QH275).

\section{Availability of data and materials}

The datasets used and/or analyzed during the current study are available from the corresponding author on reasonable request.

\section{Authors' contributions}

HX provided technical support on the study conception and supervision, and LX, SX, XW and ZJ provided technical support on cell-induced differentiation and staining. JW provided technical support on RT-qPCR and western blot analysis, and BY provided technical support on flow cytometry, colony-forming assay and statistical analysis.

\section{Ethics approval and consent to participate}

The present study was approved by the Ethics Committee of our hospital. Informed consent was obtained from all participants.

\section{Patient consent for publication}

No conflict of interest exits in the submission of this manuscript, and manuscript is approved by all authors for publication.

\section{Competing interests}

The authors have declared that no competing interests exist.

\section{References}

1. Shelerud RA: Epidemiology of occupational low back pain. Clin Occup Environ Med 5: 501-528, 2006.

2. Sehgal N, Shah RV, McKenzie-Brown AM and Everett CR: Diagnostic utility of facet (zygapophysial) joint injections in chronic spinal pain: A systematic review of evidence. Pain Physician 8: 211-224, 2005.

3. Cosenza S, Ruiz M, Toupet K, Jorgensen C and Noël D: Mesenchymal stem cells derived exosomes and microparticles protect cartilage and bone from degradation in osteoarthritis. Sci Rep 7: 16214, 2017.

4. Kumar H, Ha DH, Lee EJ, Park JH, Shim JH, Ahn TK, Kim KT, Ropper AE, Sohn S, Kim CH, et al: Safety and tolerability of intradiscal implantation of combined autologous adipose-derived mesenchymal stem cells and hyaluronic acid in patients with chronic discogenic low back pain: 1-year follow-up of a phase I study. Stem Cell Res Ther 8: 262, 2017.

5. Tian H, Wu A, Guo M, Zhang K, Chen C, Li X, Cheng X, Zhou T, Murray SS, Sun X and Zhao J: Adequate restoration of disc height and segmental lordosis by lumbar interbody fusion decreases adjacent segment degeneration. World Neurosurg 118: e856-e864, 2018.

6. Kim JS, Ali MH, Wydra F, Li X, Hamilton JL, An HS, Cs-Szabo G, Andrews S, Moric M, Xiao G, et al: Characterization of degenerative human facet joints and facet joint capsular tissues. Osteoarthritis Cartilage 23: 2242-2251, 2015.

7. Livak KJ and Schmittgen TD: Analysis of relative gene expression data using real-time quantitative PCR and the 2(-Delta Delta C(T)) method. Methods 25: 402-408, 2001.

8. Hunter DJ and Felson DT: Osteoarthritis. BMJ 332: 639-642, 2006.

9. Shuang F, Zhou Y, Hou SX, Zhu JL, Liu Y, Zhang CL and Tang JG: Indian Hedgehog signaling pathway members are associated with magnetic resonance imaging manifestations and pathological scores in lumbar facet joint osteoarthritis. Sci Rep 5: 10290, 2015.
10. Xu D, Sun Y, Bao G, Liu W, Zhu X, Cui S, Fan J and Cui Z: MMP-1 overexpression induced by IL-1beta: Possible mechanism for inflammation in degenerative lumbar facet joint. J Orthop Sci 18: 1012-1019, 2013

11. Alsalameh S, Amin R, Gemba T and Lotz M: Identification of mesenchymal progenitor cells in normal and osteoarthritic human articular cartilage. Arthritis Rheum 50: 1522-1532, 2004.

12. Poole CA: Articular cartilage chondrons: Form, function and failure. J Anat 191: 1-13, 1997.

13. Lopez-Alcorocho JM, Aboli L, Guillen-Vicente I, Rodriguez-Iñigo E, Guillen-Vicente M, Fernández-Jaén TF, Arauz S, Abelow S and Guillen-García P: Cartilage defect treatment using high-density autologous chondrocyte implantation: Two-year follow-up. Cartilage 9: 363-369, 2018.

14. Ma N, Wang H, Xu X, Wan Y, Liu Y, Wang M, Yu W, Dai Y, Peng J, Guo Q, et al: Autologous-cell-derived, tissue-engineered cartilage for repairing articular cartilage lesions in the knee: Study protocol for a randomized controlled trial. Trials 18: 519, 2017.

15. Wong CC, Chen CH, Chan WP, Chiu LH, Ho WP, Hsieh FJ, Chen YT and Yang TL: Single-stage cartilage repair using platelet-rich fibrin scaffolds with autologous cartilaginous grafts. Am J Sports Med 45: 3128-3142, 2017.

16. Elima K and Vuorio E: Expression of mRNAs for collagens and other matrix components in dedifferentiating and redifferentiating human chondrocytes in culture. FEBS Lett 258: 195-198, 1989.

17. Schnabel M, Marlovits S, Eckhoff G, Fichtel I, Gotzen L, Vécsei V and Schlegel J: Dedifferentiation-associated changes in morphology and gene expression in primary human articular chondrocytes in cell culture. Osteoarthritis Cartilage 10: 62-70, 2002.

18. Diaz-Romero J, Gaillard JP, Grogan SP, Nesic D, Trub T and Mainil-Varlet P: Immunophenotypic analysis of human articular chondrocytes: Changes in surface markers associated with cell expansion in monolayer culture. J Cell Physiol 202: 731-742, 2005.

19. Stoddart MJ, Grad S, Eglin D and Alini M: Cells and biomaterials in cartilage tissue engineering. Regen Med 4: 81-98, 2009.

20. Williams R, Khan IM, Richardson K, Nelson L, McCarthy HE, Analbelsi T, Singhrao SK, Dowthwaite GP, Jones RE, Baird DM, et al: Identification and clonal characterisation of a progenitor cell sub-population in normal human articular cartilage. PLoS One 5: e13246, 2010.

21. Hattori S, Oxford C and Reddi AH: Identification of superficial zone articular chondrocyte stem/progenitor cells. Biochem Biophys Res Commun 358: 99-103, 2007.

22. Kristjánsson $B$ and Honsawek S: Current perspectives in mesenchymal stem cell therapies for osteoarthritis. Stem Cells Int 2014: 194318, 2014.

23. Kristjánsson B, Limthongkul W, Yingsakmongkol W, Thantiworasit $\mathrm{P}$, Jirathanathornnukul $\mathrm{N}$ and Honsawek S: Isolation and Characterization of Human Mesenchymal Stem Cells From Facet Joints and Interspinous Ligaments. Spine (Phila Pa 1976) 41: E1-E7, 2016.

24. Liu LT, Huang B, Li CQ, Zhuang Y, Wang J and Zhou Y: Characteristics of stem cells derived from the degenerated human intervertebral disc cartilage endplate. PLoS One 6: e26285, 2011.

25. McAnany SJ, Ahn J, Elboghdady IM, Marquez-Lara A, Ashraf N, Svovrlj B, Overley SC, Singh K and Qureshi SA: Mesenchymal stem cell allograft as a fusion adjunct in one- and two-level anterior cervical discectomy and fusion: A matched cohort analysis. Spine J 16: 163-167, 2016.

26. Pneumaticos SG, Triantafyllopoulos GK, Chatziioannou S, Basdra EK and Papavassiliou AG: Biomolecular strategies of bone augmentation in spinal surgery. Trends Mol Med 17: 215-222, 2011.

27. Dowthwaite GP, Bishop JC, Redman SN, Khan IM, Rooney P, Evans DJ, Haughton L, Bayram Z, Boyer S, Thomson B, et al: The surface of articular cartilage contains a progenitor cell population. J Cell Sci 117: 889-897, 2004.

28. Khan IM, Williams R and Archer CW: One flew over the progenitor's nest: Migratory cells find a home in osteoarthritic cartilage. Cell Stem Cell 4: 282-284, 2009.

29. Badylak S, Obermiller J, Geddes L and Matheny R: Extracellular matrix for myocardial repair. Heart Surg Forum 6: E20-E26, 2003.

30. Olvera D, Sathy BN, Carroll SF and Kelly DJ: Modulating microfibrillar alignment and growth factor stimulation to regulate mesenchymal stem cell differentiation. Acta Biomater 64: 148-160, 2017.

31. Yang Q, Teng BH, Wang LN, Li K, Xu C, Ma XL, Zhang Y, Kong DL, Wang LY and Zhao YH: Silk fibroin/cartilage extracellular matrix scaffolds with sequential delivery of TGF- $\beta 3$ for chondrogenic differentiation of adipose-derived stem cells. Int $\mathrm{J}$ Nanomedicine 12: 6721-6733, 2017. 